\title{
THE CLOSURE OF THE REGULAR OPERATORS IN A RING OF OPERATORS
}

\author{
JACOB FELDMAN ${ }^{1}$ AND RICHARD V. KADISON
}

1. Introduction. In $[4]^{2}$ the elements of a Banach algebra are separated into various classes: the regular elements, the singular elements, the null divisors, the generalized null divisors, and so forth. These classes and their interrelations are then studied in detail. In [5] transformations between Banach spaces are studied and the classes of [4], after suitable generalization, are characterized. Among other things, it is proved in [5] that each isomorphism between a Banach space and a proper, closed, linear subspace lies in the complement of the closure of the set of regular (invertible), continuous, linear transformations of the Banach space into itself relative to the norm (uniform) topology on the set of continuous, linear transformations. Precisely what transformations lie in the closure of the set of regular transformations seems nowhere to be recorded, even in the special case where the Banach space in question is a Hilbert space. This fact would seem to have some intrinsic interest as well as possible technical usefulness.

In the following section, we describe completely those operators which lie in the closure of the set of regular operators in an arbitrary ring of operators (weakly closed, self-adjoint operator algebra on a Hilbert space)-cf. Theorem 1. Roughly speaking, this closure consists of those operators in the ring whose "essential domain" and corresponding range have equal relative co-dimension. This result is then applied to the case of factors (rings of operators whose center consists of scalar multiples of the identity) on a separable Hilbert space. It is shown, for example, that the complement of the uniform closure of the set of regular operators on a separable Hilbert space consists of those operators which can be expressed as the product of a regular operator and a partially isometric operator between subspaces whose orthogonal complements have unequal dimensions. The concluding section contains some examples which illustrate the difficulties that arise in examining the uniform closure of the set of regular operators in an arbitrary $C^{*}$-algebra (uniformly closed, self-adjoint operator algebra).

Presented to the Society, June 19, 1954; received by the editors April 9, 1954.

1 The first named author is a Fellow of The National Science Foundation.

${ }^{2}$ Numbers in brackets refer to the bibliography at the end of this paper. 
2. The closure. We introduce the notation $r(A)$ for the closure of the range of the operator $A$ and $n(A)$ for the null space of $A$. The main result is contained in:

THEOREM 1. If $R$ is a ring of operators on the Hilbert space $\mathcal{H C}$ and $G$ is the family of regular operators in $R$, then $G^{-}$, the uniform closure of $G$, consists of those operators $A$ in $R$ such that, for each $\epsilon>0$, there exists a subspace ${ }^{3} E$, belonging to $R$, containing the null space of $A$, with $\|A E\|$ $<\epsilon$ and $E$ equivalent ${ }^{4}$ in $R$ to $\mathcal{H C} \theta[A(I-E)]$.

Proof. Suppose $A$ is an operator of the type described in the statement of the theorem. Let $\epsilon>0$ be given, and choose $E$ in $R$ containing the null space of $A$ so that $\|A E\|<\epsilon$ and $E$ is equivalent to $\mathcal{F} \ominus r[A(I-E)]$. Let $A(I-E)=U_{1} H_{1}$ be the polar decomposition of $A(I-E)$ (so that $H_{1}=\left[(I-E) A^{*} A(I-E)\right]^{1 / 2}, U_{1}$ is a partial isometry from $r\left(H_{1}\right)$ to $r[A(I-E)]$, and $U_{1}, H_{1}$ lie in $R$, by [3, Lemma 4.4.1]). Now

$$
r\left(H_{1}\right)=r\left(H_{1}^{2}\right)=r\left[(I-E) A^{*} A(I-E)\right]=I-E,
$$

for $r\left(H_{1}\right)$ is $\mathfrak{H} \ominus n\left(H_{1}\right), r\left(H_{1}^{2}\right)$ is $\mathfrak{H} \ominus n\left(H_{1}^{2}\right)$; and $n\left(H_{1}\right)=n\left(H_{1}^{2}\right)$, since $H_{1}$ is positive. Moreover,

$$
r\left[(I-E) A^{*} A(I-E)\right]=\mathscr{H} \theta n\left[(I-E) A^{*} A(I-E)\right],
$$

and we shall show that $n\left[(I-E) A^{*} A(I-E)\right]=E$. Clearly $E \leqq$ $n\left[(I-E) A^{*} A(I-E)\right]$. Let $x$ be a vector in $n\left[(I-E) A^{*} A(I-E)\right]$ orthogonal to $E$, so that

$$
0=\left((I-E) A^{*} A(I-E) x, x\right)=\|A(I-E) x\|^{2}=\|A x\|^{2} ;
$$

and $x$ is in $n(A)$-hence in $E$. Thus $0=E x=x$, and $E=n\left(H_{1}^{2}\right)$. Let $U_{2}$ be a partial isometry in $R$ mapping $E$ upon $\mathfrak{H} \theta r[A(I-E)]$ (which exists by choice of $E$ ), and let $U=U_{1}+U_{2}$. Then $U$ is unitary, in $R$, and $A(I-E)=U H_{1}$. Now $U\left(H_{1}+\epsilon I\right)$ is a regular operator in $R$, and

$$
\left\|A-U\left(H_{1}+\epsilon I\right)\right\| \leqq\|A E\|+\left\|A(I-E)-U\left(H_{1}+\epsilon I\right)\right\|<2 \epsilon .
$$

Suppose now that $A$ is an operator in $R$ not of the type described in the statement of the theorem, so that there exists a constant $k>0$ such that if $E$ is a subspace of $R$ containing the null space of $A$ with $\|A E\| \leqq k$, then $E$ is not equivalent, in $R$, to $\mathfrak{F} \ominus r[A(I-E)]$. Let $A=U H$ be the polar decomposition of $A\left(H=\left(A^{*} A\right)^{1 / 2}, U\right.$ a partial

${ }^{3}$ We use the same symbol to designate a closed subspace of $\mathfrak{H C}$ and the orthogonal projection operator on this subspace.

4 For the notion of equivalence of projections, dimension function, etc., see [2] or $[3]$. 
isometry from $r(H)$ to $r(A))$. Let $E$ be the null space of the operator $(H-k I) \bigvee 0$. Then $\|A E\| \leqq k$, for $\|H E\| \leqq k$, by the Spectral Theorem, so that

$$
\|A E\|=\|U H E\| \leqq\|U\| \cdot\|H E\| \leqq k .
$$

Moreover, $E$ contains the null space of $H$ which is the null space of $A$. It follows from our hypothesis on $A$ that $E$ is not equivalent (in $R$ ) to $\mathfrak{F} \ominus r[A(I-E)]$. We note that $H(I-E) \geqq k(I-E)$, from the Spectral Theorem, so that $H$, restricted to $I-E$, has an inverse operator, on $I-E$. Let $T$ be the operator inverse to $H$ on $I-E$ and 0 on $E$. Then $T$ lies in $R$, for, if $A^{\prime}$ commutes with $R$ and $z=x+y$ with $x$ in $I-E$ and $y$ in $E$, then

$$
T A^{\prime}(x+y)=T\left(A^{\prime} H u+A^{\prime} y\right)=T\left(H A^{\prime} u+A^{\prime} y\right)=A^{\prime} u=A^{\prime} T(H u+y) ;
$$

so that $T$ commutes with $A^{\prime}$. Now $A T=U H T=U(I-E)=U_{1}$, and $U_{1}$ is a partial isometry from $I-E$ to $r[A(I-E)]$. Thus, if $A$ is a uniform limit of the regular operators $A_{n}$ in $R$, then $U_{1}(=A T)$ is a limit of the regular operators $A_{n}(T+1 / n)$. We shall show that $U_{1}$ is not a limit of regular operators in $R$. To simplify the notation, our situation is the following: $V$ is a partial isometry from a subspace $P$ to a subspace $Q$ with $I-P$ and $I-Q$ not equivalent in $R$, we must show that $V$ is not a uniform limit of regular operators in $R$. According to [2, Theorem 5.6], we can find three central projections $E, F, G$ such that $E+F+G=I$, and

$$
E(I-P) \prec E(I-Q), F(I-P) \sim F(I-Q), G(I-Q) \prec G(I-P) \text {. }
$$

The fact that $I-P$ is not equivalent to $I-Q$ tells us that $F \neq I$, so that not both $E$ and $G$ are 0 . We may assume $E$ is not 0 , for, if this is not the case, we work with $V^{*}$, so that $I-P, I-Q$ and $E, G$ interchange their roles. Of course, $V^{*}$ is a limit of regular operators in $R$ if and only if $V$ is such. If $V$ is a limit of regular operators in $R$, then $V E$ restricted to $E$ is a limit of regular operators in $R E$ restricted to $E$ (recall that $E$ is a central projection in $R$ ). Moreover, $V E$ is a partial isometry from $P E$ to $Q E$. This analysis in terms of central projections shows us that it suffices to deal with the case where $I-P$ $<I-Q$ in order to show that $V$ is not a limit of regular operators in $R$. This assumption being made, suppose that $B$ is a regular operator in $R$ with $B=K U$ the polar decomposition of $B$, and that $\|V-K U\|$ $<1 / 3$. Now $U$ is unitary, so that $\left\|V U^{*}-K\right\|<1 / 3$, and $V U^{*}$ is a partial isometry from $U P(\mathcal{H C})$ to $Q$. Of course,

$$
U(I-P) \mathfrak{H C}\langle U(I-Q)(\mathcal{H C}) \sim(I-Q),
$$


and $U(I-P)(\mathcal{F C})$, is the orthogonal complement of $U P(\mathcal{F C})$. By generalizing [1, Lemma 7] from factors to rings of operators, we conclude that $U P(\mathfrak{H C}) \cap(I-Q) \neq(0)$. In fact, $[2$, Theorem 5.4] states that for each pair of projections $E_{1}, F_{1}$ in $R$,

$$
E_{1} \cup F_{1}-F_{1} \sim E_{1}-E_{1} \cap F_{1} .
$$

Applying this with $I-Q$ for $E_{1}$ and $U P(\mathfrak{H C})$ for $F_{1}$, we see that if $(I-Q) \cap U P(\mathfrak{F})=(0)$ then

$$
(I-Q) \sim(I-Q) \cup U P(\mathcal{F})-U P(\mathcal{H C}) \leqq U(I-P)(\mathcal{H}),
$$

contradicting $U(I-P)(\mathcal{F C}) \prec(I-Q)$. Thus $U P(\mathcal{H C}) \cap(I-Q) \neq(0)$ as asserted. Let $x$ be a unit vector in the intersection of these subspaces. Then,

$$
1 / 3>\left\|V U^{*} x-K x\right\| \geqq\left\|V U^{*} x\right\|-\|K x\|=1-\|K x\|,
$$

and

$$
\|K x\|>2 / 3
$$

On the other hand,

$$
1 / 3>\left\|U V^{*}-K^{*}\right\|=\left\|U V^{*}-K\right\|
$$

so that

$$
1 / 3>\left\|U V^{*} x-K x\right\|=\|K x\|,
$$

a patent absurdity. It follows that $V$ and hence $A$ are not uniformly approximable by regular operators. In connection with the above inequalities, note that, by choice of $x, U^{*} x$ lies in $P$, so that

$$
\left\|V U^{*} x\right\|=\left\|U^{*} x\right\|=\|x\|=1 \text {; }
$$

and that $x$ lies in $I-Q$, so that $V^{*} x=0=U V^{*} x$. This completes the proof.

We apply Theorem 1 to the case where our ring $R$ is a factor on a separable Hilbert space. In this case some of the complications present in the more general situation disappear, and the characterization of the operators in the closure of the set of regular operators takes a simpler form.

Theorem 2. An operator $A$ on a separable Hilbert space $\operatorname{TC}$ is not in the closure, $G^{-}$, of the set, $G$, of regular operators on $\mathfrak{H C}$ if and only if there exists a constant $k>0$ such that $\|A x\| \geqq k$ for each unit vector $x$ in $M$, the orthogonal complement of the null space, $N$, of $A$, and dimension $(N)$ $\neq$ dimension $(\mathcal{H} \ominus r(A))$. Equivalently, $A$ is not in $G^{-}$if and only if 
$A$ is the product of a regular operator and a partially isometric operator between subspaces of unequal co-dimension.

Proof. If $A$ does not satisfy the conditions stated in the theorem, then either dimension $(N)=\operatorname{dimension}(\mathfrak{H C} \ominus r(A))$ or there is no positive constant such as $k$. If dimension $(N)=\operatorname{dimension}(\mathcal{H} \ominus r(A))$, we can choose $N$ as our $E$ in Theorem 1 (for all $\epsilon>0$ ); so that $A$ is in $G^{-}$. Suppose then that dimension $(N) \neq$ dimension $(\mathcal{H} \ominus r(A))$. We may assume that dimension $(N)<\operatorname{dimension}(\mathcal{H} \ominus r(A))$, for, otherwise, we deal with $A^{*}$, observing that $N=\mathscr{H} \ominus r\left(A^{*}\right)$ and $\mathscr{H} \ominus r(A)$ $=n\left(A^{*}\right)$. Since $\mathfrak{T C}$ is separable, this assumption implies, in particular, that dimension $(N)$ is finite. Our hypothesis about $A$ implies that there is no constant $k$ such as described in the statement of the theorem. Thus, for each $\epsilon>0$, one can find a unit vector $x$ in $M$ such that $\|A x\|<\epsilon$. This assumption on $A$ implies that the spectral projection, $E_{1}$, for $A^{*} A$ corresponding to the interval $\left[0, \epsilon^{2}\right]$ (and, so, for every interval $[0, \beta], \beta>0)$ contains $N$ properly. In fact, $E_{1}$ contains $N$, and

$$
\epsilon^{2}>\left(A^{*} A x, x\right) \geqq\left(A^{*} A\left(I-E_{1}\right) x, x\right) \geqq \epsilon^{2}\left(\left(I-E_{1}\right) x, x\right) ;
$$

so that

$$
1>(x, x)-\left(E_{1} x, x\right)=1-\left(E_{1} x, x\right),
$$

and

$$
\left(E_{1} x, x\right)>0=(N x, x) .
$$

It follows that $E_{1}$ is infinite-dimensional, for, otherwise, $A^{*} A$ has a finite number of points less than $\boldsymbol{\epsilon}^{2}$ in its spectrum; and we can certainly find $\beta, \epsilon^{2}>\beta>0$, such that the spectral projection for $A^{*} A$ corresponding to the interval $[0, \beta]$ is equal to $N$. We take $E_{1}$ as $E$ of Theorem 1 corresponding to the given $\epsilon$, and note that $E_{1}$, having infinite dimension, is equivalent to $\operatorname{He} \theta r\left[A\left(I-E_{1}\right)\right]$. In fact, with $A=W\left(A^{*} A\right)^{1 / 2}$ the polar decomposition of $A, W$ maps $r\left[\left(A^{*} A\right)^{1 / 2}\right]$ $=r\left[\left(A^{*} A\right)\right]=\mathfrak{H} \ominus N$ isometrically upon $r(A)$, and $\left(A^{*} A\right)^{1 / 2}\left(I-E_{1}\right)$ $\geqq \epsilon\left(I-E_{1}\right)$; so that $\left(A^{*} A\right)^{1 / 2}$ maps $\left(I-E_{1}\right)$ upon itself. Thus $r\left[A\left(I-E_{1}\right)\right]=r\left[W\left(I-E_{1}\right)\right]$, and $r\left[W\left(E_{1}-N\right)\right]$ is infinite-dimensional. But $r\left[W\left(E_{1}-N\right)\right]$ is orthogonal to $r\left[W\left(I-E_{1}\right)\right]$, since $E_{1}-N$ is orthogonal to $I-E_{1}$ and $W$ is isometric on $I-N$. Thus $\mathcal{H C}$ $\ominus r\left[W\left(I-E_{1}\right)\right]=\mathcal{H} \ominus r\left[A\left(I-E_{1}\right)\right]$ is infinite-dimensional and, therefore, equivalent to $E_{1}$ as asserted. Moreover,

$$
\left\|A E_{1}\right\|^{2}=\left\|E_{1} A^{*} A E_{1}\right\|=\left\|A^{*} A E_{1}\right\| \leqq \epsilon^{2},
$$

so that $A$ is in $G^{-}$. 
We must show, now, that if $A$ is in $G^{-}$then $A$ does not satisfy the conditions of the statement of the theorem. In fact, by Theorem 1 , given $\epsilon>0$, there exists a space $E$ containing $n(A)$ such that $\|A E\|$ $<\epsilon$, with $E$ equivalent to $\mathcal{F} \ominus r[A(I-E)]$. If there is a constant such as the $k$ of the statement of the theorem, for $A$, then, choosing $\epsilon<k$, we see that the corresponding $E$ must equal $n(A)$ (for, if $E$ contains $n(A)$ properly, there is a unit vector $x$ in $E-n(A)$, hence in $\mathcal{H C} \ominus n(A)$, such that $\|A x\|=\|A E x\|<\epsilon<k$, contradicting the choice of $k)$. But then $n(A)$ is equivalent to $\mathfrak{H} \theta r[A(I-n(A))]=\mathfrak{H} \theta r(A)$. In any event, if $A$ is in $G^{-}$, then $A$ does not satisfy the conditions of the statement of the theorem.

We prove next that an operator not in $G^{-}$is the product of a regular operator and a partially isometric operator whose initial and final spaces have unequal co-dimension, and that, conversely, such a product does not lie in $G^{-}$. Suppose, first, that $W$ is a partially isometric operator whose initial and final spaces have unequal co-dimension, and let $T$ be a regular operator. If $T W$ is the uniform limit of the regular operators $A_{n}$, then $W$ is the uniform limit of the regular operators $T^{-1} A_{n}$. But, from the first portion of the proof, $W$ is certainly not in $G^{-}$, so that $T W$ is not in $G^{-}$. Suppose, now, that $A$ is not in $G^{-}, A$ has null space $N$ with orthogonal complement $M$. We know that there exists a $k>0$ such that $\|A x\| \geqq k$ for each unit vector $x$ in $M$ and that dimension $(N) \neq$ dimension $(\mathcal{H} \ominus r(A))$. Now $r\left(A^{*} A\right)$ is $M$ and $A^{*} A$ has an inverse on $M$ (since $\left(A^{*} A x, x\right)=\|A x\|^{2} \geqq k^{2}$ for each unit vector $x$ in $M)$. Thus $\left(A^{*} A\right)^{1 / 2}$ has an inverse $T^{\prime}$ on $M$. Let $A=W\left(A^{*} A\right)^{1 / 2}$ be the polar decomposition of $A, W$ a partially isometric operator with initial space $M$ and final space $r(A)$. If we define $T$ to be $T^{\prime}$ on $M$ and the identity on $N$, we have that $T$ is a regular operator, and $A T=W$; so that $A=W T^{-1}$. Since $A$ is not in $G^{-}, W$ is not in $G^{-}$, and it follows at once, from Theorem 1 , that the co-dimensions of the initial and final spaces of $W$ are unequal.

Theorem 2 is valid for factors of type $I_{\infty}$ where "co-dimension" is replaced by "relative co-dimension," since the property of being a product of a regular operator and a partial isometry of the described type is algebraic (when relative dimension replaces dimension). It is immediate, from Theorem 1 , that each operator, $A$, in a factor of type $\mathrm{II}_{1}$ is the uniform limit of regular operators in the factor (take as $E$ the null space of $A$ ). The result for factors of type $\mathrm{II}_{1}$ also follows quite easily from the fact that each operator in the factor is the product of a unitary operator and a positive operator in the factor. For factors of type III on a separable space, the complement of the uniform closure of the regular operators consists of those operators which are the product of a regular operator and a partial isometry 
whose initial and final spaces are the whole space and some proper closed subspace, in the factor, respectively, and their adjoints. In fact such an operator is not approximable by regular operators, since the partial isometry is not. Suppose $A$ is an operator with null space $N$ and $M=\mathfrak{H} \ominus N$, in a factor of type III. If both $N$ and $\mathfrak{H C} \theta r(A)$ are nonzero, then they are infinite hence equivalent, and $A$ is approximable by regular operators, by Theorem 1 (take $E$ to be $N$ for all $\epsilon>0$ ). We assume $N=(0)$ and $\mathfrak{H C} \ominus r(A) \neq(0)$ (otherwise deal with $A^{*}$ ). Let $A=W\left(A^{*} A\right)^{1 / 2}$ be the polar decomposition of $A$. Then $W$ has $\mathcal{H C}$ as initial space (the closure of the range of $\left(A^{*} A\right)^{1 / 2}$ ) and $r(A)$ (a proper closed subspace) as final space. If for each $\epsilon>0$ the spectral projection $E^{\prime}$ for $\left(A^{*} A\right)^{1 / 2}$ corresponding to the interval $[0, \epsilon]$ is nonzero, then $E^{\prime}$ is infinite and will serve as the $E$ of Theorem 1 for $\epsilon$. In this case, $A$ is again approximable by regular operators in the factor, so that we may assume that $E^{\prime}=0$ for some $\epsilon>0$. But then $\left(A^{*} A\right)^{1 / 2}$ is invertible, and the polar decomposition for $A$, noted above, represents $A$ in the desired form.

3. Some examples. In the preceding section, we described the uniform closure of the set of regular operators in a ring of operators relative to that ring of operators. It is natural to ask, at this point, whether or not some such description could be given to the uniform closure of the set of regular operators in an arbitrary $C^{*}$-algebra. The fact that the containing algebra is only uniformly closed seems to introduce topological difficulties not present in the case of a weakly closed algebra. Moreover, there seems to be little connection between the closure of the regular operators in a $C^{*}$-algebra and the closure of the regular operators in the ring of operators generated by this $C^{*}$-algebra (i.e., its weak closure).

The difficulties just mentioned occur even in the case of abelian $C^{*}$-algebras with identity (function rings on compact-Hausdorff spaces) as we shall illustrate in the following examples. First, however, we note the situation for abelian rings of operators. Each operator $A$ in an abelian ring of operators is uniformly approximable by regular operators in the ring. In fact, $A$ commutes with $A^{*}$, so that $A$ is normal, and $N$, the null space of $A$, is the null space of $A^{*}$ (for a normal operator, $A,\|A x\|=\left\|A^{*} x\right\|$ for each vector $x$ ). Thus, if $M$ is the orthogonal complement of $N$, then $M$ is the closure of the range of $A$, and we may take $N$ as $E$ in Theorem 1 for each $\epsilon>0$.

Examples 1 and 2, following, illustrate the way in which the connectivity properties of the underlying compact-Hausdorff space influence approximability by regular operators.

Example 1. As our Hilbert space $\mathfrak{F}$, we take $L_{2}(0,1)$, and, as our 
algebra, $A$, we take the family of all operators $T_{f}$ defined on each function $g$ in $L_{2}(0,1)$ by $T_{f}(g)=f g, f$ real and continuous on $[0,1]$. The algebra $A$ is an abelian $C^{*}$-algebra over the reals. The spectrum of $T_{f}$ is the range of values of $f$. It is not difficult to see that $T_{f}$ is not approximable by regular operators in $\mathcal{A}$ if and only if 0 is an inner point of the spectrum of $T_{f}$ (relative to the real line). On the other hand, the weak closure of $\mathcal{A}$ is abelian, so that each operator in $A$ is approximable by regular operators in this closure.

EXAmple 2. As our Hilbert space $\mathfrak{H}$, we take $L_{2}$ functions on the closed unit disk in the complex plane, and as $A$, the algebra of operators defined formally as in Example 1. Simple homotopy considerations show that an approximant to $T_{z}$ (multiplication by $z$ ) must have 0 in its spectrum; i.e., $T_{z}$ is not approximable by regular operators in $\mathcal{A}$.

Example 3. As our Hilbert space, $\mathfrak{F}$, we take $L_{2}$ functions on the upper half of the unit disk in the complex plane; more precisely, those complex numbers $z=\alpha \exp (i \theta)$ such that $0 \leqq \alpha \leqq 1$ and $0 \leqq \theta \leqq \pi$, and again as $\mathcal{A}$ the algebra of multiplications by continuous complexvalued functions. As an indication that more is involved than 0 being an inner point of the spectrum, we exhibit an operator in $A$ whose spectrum is, in fact, the unit disk, which is, nonetheless, uniformly approximable by regular operators in $\mathcal{A}$. Define $f$ by $f(\alpha \exp (i \theta))$ $=\alpha \exp (i 2 \theta)$. The range of $f$ is the unit disk, so that the spectrum of $T_{f}$ is the unit disk. On the other hand, $T_{f}$ is approximable by regular operators in $\mathcal{A}$. In fact, given $\epsilon>0$, we define $f_{1}$ as follows: $f_{1}(\alpha \exp (i \theta))=\alpha \exp (i(2 \theta(1-\epsilon / \pi)+\epsilon))$. There is no difficulty in verifying that $\left\|f_{1}-f\right\|<\epsilon$, so that $\left\|T_{f_{1}}-T_{f}\right\|<\epsilon$. Moreover, if $\beta>0$, then $\beta$ is not in the range of $f_{1}$, and therefore not in the spectrum of $T_{f_{1}}$. Thus $T_{f_{1}}-\beta I$ is regular, in $\mathcal{A}$, and, for small $\beta,\left\|T_{f_{1}}-\beta I-T_{f}\right\|$ $<\epsilon$, establishing the desired result.

\section{BibliogRAPHY}

1. R. Kadison, Infinite unitary groups, Trans. Amer. Math. Soc. vol. 72 (1952) pp. 386-399.

2. I. Kaplansky, Projections in Banach algebras, Ann. of Math. vol. 53 (1951) pp. 235-249.

3. F. Murray and J. von Neumann, On rings of operators, Ann. of Math. vol. 37 (1936) pp. 116-229.

4. C. Rickart, The singular elements of a Banach algebra, Duke Math. J. vol. 14 (1947) pp. 1063-1077.

5. B. Yood, Transformations between Banach spaces in the uniform topology, Ann. of Math. vol. 50 (1949) pp. 486-503.

Columbia University 